\title{
Biodiversidad, morfometría y alimentación de los cangrejos del género Callinectes (Decapoda: Portunidae) en Santiago de Cuba
}

\author{
Liliana Gómez Luna ${ }^{1}$, Antonio Sosa Montano ${ }^{2}$, Isabel Moreno Castillo 3 \& Abdiel Jover Capote ${ }^{4}$ \\ 1. Laboratorio de Ecotoxicología, Centro Nacional de Electromagnetismo Aplicado, Universidad de Oriente. Santiago \\ de Cuba, Ave. de las Américas Esquina I, CP 90900; lilianag@cnea.uo.edu.cu \\ 2. Departamento de Bioelectromagnetismo, Centro Nacional de Electromagnetismo Aplicado, Universidad de Oriente, \\ Santiago de Cuba, Ave. de las Américas Esquina I, CP 90900; antonio@cnea.uo.edu.cu \\ 3. Departamento de Biología, Facultad de Ciencias, Universidad de Islas Baleares, España; isabel.moreno@uib.es \\ 4. Departamento de Biología, Facultad de Ciencias Naturales, Universidad de Oriente; abdiel@cnt.uo.edu.cu
}

Recibido 12-IX-2008. Corregido 18-XI-2008. Aceptado 16-XI-2008.

\begin{abstract}
Biodiversity, morphometry and diet of Callinectes crabs (Decapoda: Portunidae) in Santiago de Cuba. On the basis of fishery intensity and proximity to river mouth, a total of 257 swimming Callinectes crabs were collected from March 2007 to April 2008 in eight localities. Captures were made with hanging nets, to a maximal depth of $1.5 \mathrm{~m}$, establishing a top time of $2 \mathrm{hr}$. The genus was widely distributed, and it is represented at least by four species: C. sapidus, C. similis, C. rathbunae and C. larvatus, the last two not included in the last list of Cuban crustaceans (Crustacea: Decapoda). The size and shape of the gonopods were very useful as taxonomical criteria, considering the prevalence of males. The most abundant species were C. sapidus (47.08\%), and C. similis (30.35\%). C. rathbunae, which was identified at $75 \%(6)$ of the localities, showed a better distribution, followed by C. sapidus, which appears in the $63 \%(5)$. Sex rate $\left(\mathrm{R}_{\mathrm{s}}\right)$ by species (male:female) was 6.20 for C. rathbunae, 3.58 for C. similis, 1.40 for C. larvatus and 0.40 for $C$. sapidus. The most abundant species had a lower $\mathrm{R}_{\mathrm{s}}$ value. Morphometrical analysis and weight allowed us to know the average carapace width $(\mathrm{CW})$ and weight $(\mathrm{W})$. C. sapidus had an average $\mathrm{CW}=110.57 \mathrm{~mm}(\mathrm{DS} 21.55, \mathrm{n}=121)$ and $\mathrm{W}=84.46 \mathrm{~g}(\mathrm{SD} 43.25, \mathrm{n}=$ 121); C. rathbunae a $\mathrm{CW}=115.50 \mathrm{~mm}(\mathrm{DS} 14.94, \mathrm{n}=36)$ and $\mathrm{W}=140.44 \mathrm{~g}(\mathrm{DS} 55.02, \mathrm{n}=36)$; C. larvatus a $C W=76.04 \mathrm{~mm}(\mathrm{DS} 10.88, \mathrm{n}=22)$ and $\mathrm{W}=31.70 \mathrm{~g}(\mathrm{DS} \mathrm{14.67}, \mathrm{n}=22)$; and $C$. similis had the minimal parameter values, with a $\mathrm{CW}=59.77 \mathrm{~mm}(\mathrm{DS} 14.09, \mathrm{n}=78)$ and $\mathrm{W}=13.80 \mathrm{~g}(\mathrm{DS} 10.00, \mathrm{n}=78)$. These are the first records of the coastal crabs in the area. All values are lower than in previous reports. The largest individuals (CW $>140 \mathrm{~mm}$ : C. rathbunae and $C$. sapidus) were captured in localities with adequate environmental characteristics. The stomach content suggests nine dietary categories, mainly fishes and macroalgae. The genus Callinectes has a diversified trophic spectrum. These crabs eat the available food, but they have a preference for the most abundant items. Rev. Biol. Trop. 57 (3): 671-686. Epub 2009 September 30.
\end{abstract}

Key words: Callinectes, C. sapidus, C. similis, C. rathbunae y C. larvatus, crab, Santiago de Cuba.

Los cangrejos portúnidos constituyen un grupo faunístico conspicuo, distribuido ampliamente en ambientes tropicales y subtemplados del Atlántico Occidental. Dentro de este grupo de crustáceos se encuentra el género Callinectes Stimpson 1860, ampliamente estudiado, sobre todo en la costa este de los Estados Unidos y el Golfo de México, destacando los trabajos realizados acerca de $C$. sapidus y $C$. rathbunae, especies sobre las que se ha constituído desde hace varios años la pesquería en la región (Van Engel 1958a,1958b, Pounds 1964, Tagatz 1965, 1968).

El género incluye 16 especies ampliamente distribuidas en los neotrópicos (Williams 1974). En la región del Caribe y del Golfo del México se han identificado C. rathbunae Contreras 1930, C. sapidus Rathbun 1896 
(subespecie: C. acutidens Voss 1976), C. similis Williams 1966, C. exasperatus Gerstaecker 1856, C. larvatus Ordway 1863, C. marginatus A. Milne Edwards 1861 y C. ornatus Ordway 1863, además de C. bocourti A. Milne Edwards 1879 y $C$. danae Smith 1869; mientras que $C$. maracaiboensis Taissoun 1969 ha sido identificada hasta el momento sólo en el sistema estuárico del Lago Maracaibo, aunque quizás pueda tener una distribución más amplia.

Los patrones de distribución parecen estar condicionados por factores como la salinidad, sobre todo en el Caribe, dentro y entre islas (Norse 1972, 1977, 1978), la protección, alimentación, maduración, temperatura, reclutamiento y eventos reproductivos, según señalan Ortiz et al. (2007), sin restar importancia a la composición y textura del sedimento (Adkins 1972), la extensión del estuario y la cercanía a ecosistemas productivos como los manglares, por su función como proveedores de refugio y alimento (Buchanan y Stoner 1988).

La especie más tratada dentro del género por su abundancia y representatividad en ecosistemas tropicales de aguas estuarinas y someras es C. sapidus; esta forma parte de las pesquerías de varios países (Ramírez y Hernández 1988). En Cuba, Baisre (2004) explica que de manera general existen tres especies de cangrejos con interés comercial, representando en conjunto el $3.1 \%$ de las capturas pesqueras nacionales, entre los que está $C$. sapidus. Asimismo, se han identificado poblaciones comerciales de jaibas, en la costa norte y en la suroriental, hacia la desembocadura del río Cauto, donde son abundantes (Valdés et al. 1994).

Las especies identificadas en ecosistemas costeros de Cuba, según los listados actualizados de decápodos (Lalana y Ortiz 2000) son: $C$. sapidus. C. ornatus, C. danae, C. marginatus, C. exasperatus y $C$. bocourti; siendo una de las zonas más estudiadas la del Golfo de Batabanó (Martínez-Iglesias y Gómez 1986).

Ortiz et al. 2007 refieren que son muchas las investigaciones realizadas sobre biología, patrones de distribución, reproducción, manejo y aprovechamiento pesquero de especies del género Callinectes. Es también importante la contribución de Norse (1972, 1977, 1978), Moncada y Gómez (1980), Rodríguez (1982), Román-Contreras (1986 a, 1986b) Rosas y Lázaro-Chávez (1986), Markham et al. (1987), García-Montes et al. (1988), Buchanan y Stoner (1988), Rocha-Ramírez et al. (1992), Rosas y Sánchez (1994), Ramos et al. (1998) y Criales et al. (2002) en cuanto a morfometría, patrones alimentarios, fecundidad, migraciones, ecología, etología, físiología, y más reciente los estudios sobre filogenia molecular de Robles et al. (2007), de composición bioquímica (Tsai et al. 1984) y calidad proteica (Küçükgülmez y Çelik 2008) han enriquecido el conocimiento sobre el género. Sin embargo, los estudios locales cobran relevancia ante la marcada interacción de factores químicos, físicos y biológicos que condiciona la distribución y abundancia de estas especies estuarinas, y su calidad como alimento.

Este estudio examina la identificación de especies del género Callinectes en el litoral de Santiago de Cuba, su distribución, abundancia, morfometría, características relevantes de los hábitats y principales categorías dietéticas, en una zona donde no hay antecedentes de investigaciones sobre biología, ecología o manejo de pesquerías de estos organismos, y en la que los estudios carcinológicos no han tenido hasta la fecha un lugar relevante.

\section{MATERIALES Y MÉTODOS}

Área de estudio: Este estudio fue desarrollado durante el período comprendido entre marzo de 2007 a abril de 2008. Se realizaron capturas de especies del género Callinectes en ocho estaciones preestablecidas en el litoral del municipio Santiago de Cuba, considerando sitios de pesca preexistentes, donde habitualmente operan grupos de pescadores y sitios próximos a esteros o desembocaduras de ríos, donde no se han establecido pesquerías por no ser de fácil acceso.

El municipio Santiago de Cuba está localizado en la costa sudeste de la isla de Cuba en una zona de intensa actividad marítima y una 
región que se caracteriza por un ambiente marino frágil. La línea costera del municipio tiene una extensión de $205 \mathrm{~km}$ y está ubicada a todo lo largo del sistema montañoso de la Sierra Maestra. Los límites de este estudio comprenden la zona costera desde Bahía Cabañas hasta la desembocadura del río Baconao, lo que representa el $83.11 \%$ de la línea costera del municipio.

El espacio costero marino es muy diverso; incluye bahías, playas, ensenadas, estuarios, costas de fondos rocosos; cayos, arrecifes en parches, así como humedales y pastos marinos (Gómez et al. 2001). La presencia de una gran terraza cársica, implica la existencia de costas abrasivas con playas expuestas, que predominan hacia el Este. En toda la franja costera las principales actividades son la pesca, tanto comercial como recreativa y de subsistencia; así como el turismo. Existe además actividad portuaria y la práctica de deportes náuticos en la bahía de Santiago de Cuba, principal accidente geográfico. De forma general, los espacios costeros son muy usados por las comunidades tanto para el esparcimiento como para desarrollar actividades que sostienen la economía familiar.

En la época de estudio destacan dos estaciones claramente definidas, denominadas período lluvioso, de mayo a octubre y el periodo de seca, de noviembre a abril.

Durante este estudio las temperaturas máximas se registraron en julio de $2007\left(29.8^{\circ} \mathrm{C}\right)$ y las mínimas en diciembre de $2007\left(25.4^{\circ} \mathrm{C}\right)$. Respecto a las precipitaciones el comportamiento fue muy irregular, registrándose valores mínimos en enero de 2008 (30.3 mm) y julio de 2007 (37.6 mm) y máximos en octubre de 2007 (573.9 mm). En 2008 los valores acumulados de precipitaciones hasta marzo fueron superiores a los reportados para el mismo período de 2007. La humedad relativa estuvo entre 67 y $83 \%$, según datos del Centro Meteorológico Provincial.

Sitios de captura: Se definen ocho sitios: 1. Punta Sal, ubicado en el interior de la Bahía de Santiago de Cuba (20¹'0.22” N-7551'44.02"
W), 2. Aguadores, sitio de playa ubicado en los 1958'5.36" N-7549'28.30” W, 3. Sardinero, sitio de playa ubicado en los $19^{\circ} 57^{\prime} 42.07^{\prime \prime}$ N-75 47’2.20” W, 4. Siboney I, estero ubicado en la playa homónima sin conexión física con la playa la mayor parte del año (1957’36” N -7542’14.76” W), 5. Siboney II, canal construido en la desembocadura del río Carpintero (1957’31.37" N-7542'9.12" W) hacia la playa Siboney, 6. Juraguá, estero ubicado en la playa homónima con salida al mar (1956’23.25” N-75'40’23.39” W), 7. Daiquirí, estero sin conexión física con la playa homónima (1955’27.91” N-75³8’37.30” W), y 8 . Baconao, ubicado en la desembocadura del río Baconao (1954’8.81” N-75²6’49.30” W) hacia la playa homónima.

\section{Caracterización de los sitios de captura:} Los sitios fueron caracterizados considerando indicadores cuantitativos como: salinidad (ups), medida con un refractómetro de mano; $\mathrm{pH}$, medido con un $\mathrm{pH}$-metro PRACITRONIC MV88; número total de individuos capturados; proporción de machos y hembras $\left(\mathrm{R}_{\mathrm{s}}\right)$; peso promedio en $\mathrm{g}(\mathrm{W})$ de los ejemplares capturados; talla promedio en $\mathrm{mm}$ (AC); número de pescadores por sitio y número de especies identificadas, e indicadores cualitativos: tipo de ecosistema (bahía, playa, estero, desembocadura de río) y características de los fondos (areno-fangoso, fangoso, arenoso) (Cuadro 1). Para cada sitio se determinó además, el índice de dominancia de Brown para la especie mejor representada, considerando la captura total.

Capturas: Se realizaron cuatro capturas en cada una de las ocho estaciones en marzo, julio y noviembre de 2007 y marzo-abril de 2008. Estas se llevaron a cabo entre las 5:00 y las 7:00 am, teniendo en cuenta que según la experiencia local, las capturas son máximas entre las 5 y 10 a.m. Si bien es conocido que los cangrejos nadadores siguen ciclos diarios en los cuales durante el día merodean por los fondos y emergen en la noche, considerar la experiencia de los pescadores locales fue una premisa de esta investigación. Se utilizaron 


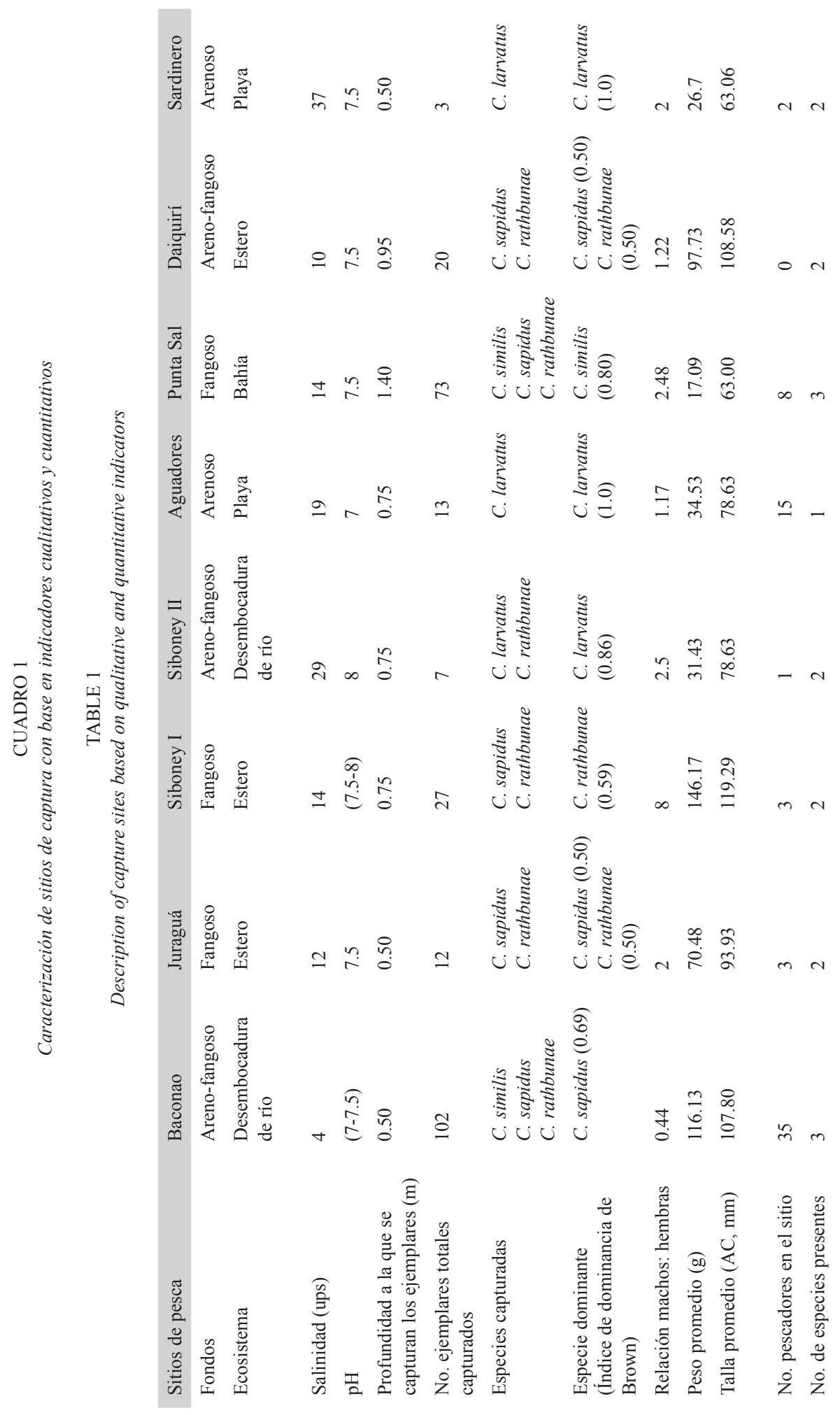


aros jaiberos de $50 \mathrm{~cm}$ de diámetro y una luz de malla de $2 \mathrm{~cm}$, estableciendo un límite temporal de captura de $2 \mathrm{hr}$ por estación. Los aros jaiberos se han usado tradicionalmente por los pescadores de jaibas en esta zona de Cuba, con gran efectividad, si bien se utilizan otras artes como el cordel, palangre, nasa, vara o pincho, chinchorro y atarraya; no obstante, considerando las características de los fondos en las estaciones seleccionadas y la profundidad de estas, además, para ejercer una menor presión de captura, se utilizaron aros jaiberos.

Las hembras ovígeras e individuos con daños en las espinas laterales, se excluyeron de la captura. Una vez capturadas, las jaibas se preservaron "in situ" en jaulas sumergidas hasta su traslado inmediato al laboratorio. Para disminuir riesgos de digestión post-captura se conservaron a $-10^{\circ} \mathrm{C}$.

Se realizaron entonces las clasificaciones y análisis correspondientes a cada ejemplar.

Identificación y caracterización de especímenes: Cada uno de los ejemplares fue sexado, identificado y caracterizado; en el caso de los machos se analizó el tamaño y diseño de los gonopodios, con la ayuda de una lente estereoscópica y un pie de rey digital Caliper con $0.01 \mathrm{~mm}$ de precisión.

Para la identificación taxonómica se consideraron las claves dicotómicas de William (1974, 1984), las modificadas por Abele y Kim (1986); los criterios de Gore y Grizzle (1974), Hendricks (1984), Martínez-Iglesias y Gómez (1986). Fueron identificados todos los ejemplares capturados $(n=257)$, clasificados por especie y sexados según Hendricks (1984, 1995a, 1995b), considerando además los criterios de Carmona-Suárez y Conde (2002) para sexar juveniles. Ejemplares testigos fueron depositados en la Colección de Crustáceos del Centro Oriental de Ecosistemas y Biodiversidad (BIOECO) con acrónimo BSCC.

Análisis morfométricos y pesaje: Se realizaron determinaciones de anchura (AC) y longitud del carapacho (LC), siguiendo las recomendaciones biométricas de Moncada y
Gómez (1980), William (1984) y CarmonaSuárez y Conde (2002), así como la altura del área metagástrica (h) en mm, utilizando un pie de rey digital Caliper con $0.01 \mathrm{~mm}$ de precisión. El peso de cada individuo (W) fue registrado utilizando para su determinación una balanza digital con $0.1 \mathrm{~g}$ de precisión.

Proporción de sexos: Se calculó la proporción de sexos $\left(\mathrm{R}_{\mathrm{s}}, \mathrm{O}^{\mathrm{T}}\right.$ : $\left.q\right)$ para cada especie y por sitio de captura.

Análisis cualitativo de contenido estomacal: Se realizaron disecciones según la metodología estándar de Dittel (1993). La selección de las categorías dietéticas se realizó según Paul (1981) y Branco et al. (2002) con algunas modificaciones. Se realizaron análisis minuciosos del contenido estomacal con ayuda de una lupa estereoscópica, y observaciones al microscopio óptico del líquido estomacal.

Se consideraron nueve categorías alimentarias: peces, macroalgas, crustáceos, excluyendo a los anfípodos, que se trataron como categoría aparte; moluscos bivalvos, gasterópodos, plantas vasculares, granos de arena y sedimento. Se establece además la categoría de material altamente digerido (MAD) para designar al material imposible de identificar por su grado avanzado de digestión. Este análisis fue realizado al $100 \%$ de los ejemplares capturados $(\mathrm{n}=275)$; sin embargo, como el estudio proyectado fue básicamente cualitativo, se consideró la presencia de una categoría si esta aparecía en el $80 \%$ de los estómagos analizados, para cada especie y por cada sitio. Para determinar su importancia en el área de estudio se calculó la frecuencia de aparición de cada una de estas categorías.

Análisis estadísticos: Las diferencias entre las tallas por especie fueron comparadas usando el test $t$ de Student de dos colas para comparación de dos medias. La normalidad de los datos fue corroborada con las bondades del test de Kolmogorov-Smirnov.

En el caso del análisis del contenido estomacal se utilizó la estadística descriptiva, 
utilizando la frecuencia de presencia, para evaluar la importancia de las diferentes categorías dietéticas.

\section{RESULTADOS}

Caracterización de los sitios de captura: De manera general los sitios resultaron ser muy heterogéneos (Cuadro 1). Callinectes ocupa una variedad de hábitats en el litoral del municipio, la mayoría de ellos estuáricos; sólo dos fueron playas (Sardinero y Aguadores), predominando en sentido general hábitats de fondos fangosos y areno-fangosos.

El pH varió en un intervalo de 7 a 8 u para todas las estaciones; sin embargo, existieron fluctuaciones importantes de salinidad, con una variación entre 4 y 39 ups.

De todos los sitios, en Baconao $(n=102)$ y Punta Sal $(n=73)$ se realizaron las mayores capturas; en estos aparecen tres de las cuatro especies identificadas (C. sapidus, C. rathbunae, $C$. similis); en el resto sólo se identificaron una o dos especies.

De forma general hay prevalencia de machos en todos los sitios, excepto en Baconao.

Las mayores tallas promedio se registraron en Siboney I (AC: $119.29 \mathrm{~mm}$ ), sitio donde la relación $\mathrm{R}_{s}$ fue mayor (8).

La profundidad a la que la captura fue efectiva varió por sitio entre 0.50 y $1.4 \mathrm{~m}$.

Respecto al número de pescadores promedio encontrados por sitio en las diferentes capturas, Baconao fue el sitio más concurrido (35), seguido por Aguadores (15) y en menor medida, Punta Sal (8). En el resto, la presencia de pescadores fue ocasional.

Los sitios de mayor riqueza observada (tres especies) fueron: Baconao y Punta Sal, los que también presentaron mayor disponibilidad de alimentos, una rica ictiofauna con abundancia de jóvenes de varias especies, lechos de macroalgas y excelentes condiciones como refugio, si bien las características de los fondos son diferentes. Los sitios de menor diversidad, donde sólo se identificó una especie (C. larvatus) fueron las playas de Aguadores y Sardinero.
Respecto a la dominancia, en Aguadores, Siboney II y Sardinero la especie dominante fue C. larvatus; en Baconao C. sapidus; en Siboney I fue $C$. rathbunae; en Punta Sal resultó ser $C$. similis; para Daiquirí y Juraguá, esteros con valores similares de salinidad, aparecen iguales proporciones de $C$. sapidus y C. rathbunae en las diferentes capturas realizadas.

En general, la dominancia de las especies no varió significativamente en las diferentes capturas, para cada uno de los sitios $(\mathrm{p}<0.05)$.

Abundancia y distribución de especies: El género resultó estar ampliamente distribuido. Se capturaron 257 individuos pertenecientes a cuatro especies: C. sapidus, C. rathbunae, C. similis y C. larvatus. Es importante señalar que $C$. similis y $C$. rathbunae no están incluidas en la última lista publicada de crustáceos cubanos.

La cantidad total de individuos capturados en cada uno de los sitios fue diferente, así como la distribución de especies (Cuadro 1). Las mayores capturas se realizaron en Baconao $(\mathrm{n}=$ 102) y Punta $\mathrm{Sal}(\mathrm{n}=73)(\mathrm{p}<0.05)$ y las mínimas en Sardinero $(n=3)$ y Siboney II $(n=7)$.

Las especies más abundantes fueron $C$. sapidus $(47.08 \%, \mathrm{n}=121)$ y $C$. similis $(30.35 \%$, $\mathrm{n}=78$ ).

C. rathbunae fue la más ampliamente distribuida, identificándose en el 75\% (6) de los sitios de captura, seguida por $C$. sapidus, que aparece en el 63\% (5).

La especie menos abundante fue C. larvatus $(8.5 \%, \mathrm{n}=22)$; sólo fue capturada en los sitios cuyos valores de salinidad estuvieron por encima de 19 ups (Fig. 1).

Desde el punto de vista taxonómico la longitud y diseño de los gonopodios fue un elemento importante para la identificación de especies, considerando la prevalencia de machos (Fig. 2).

C. rathbunae presenta los gonopodios de mayor longitud, seguidos por los de C. sapi$d u s$. Ambos difieren en su diseño, siendo los de la primera sinuosamente curvados, con un punto de cruzamiento hacia el ápice, mientras que los de C. sapidus convergen en un tramo 


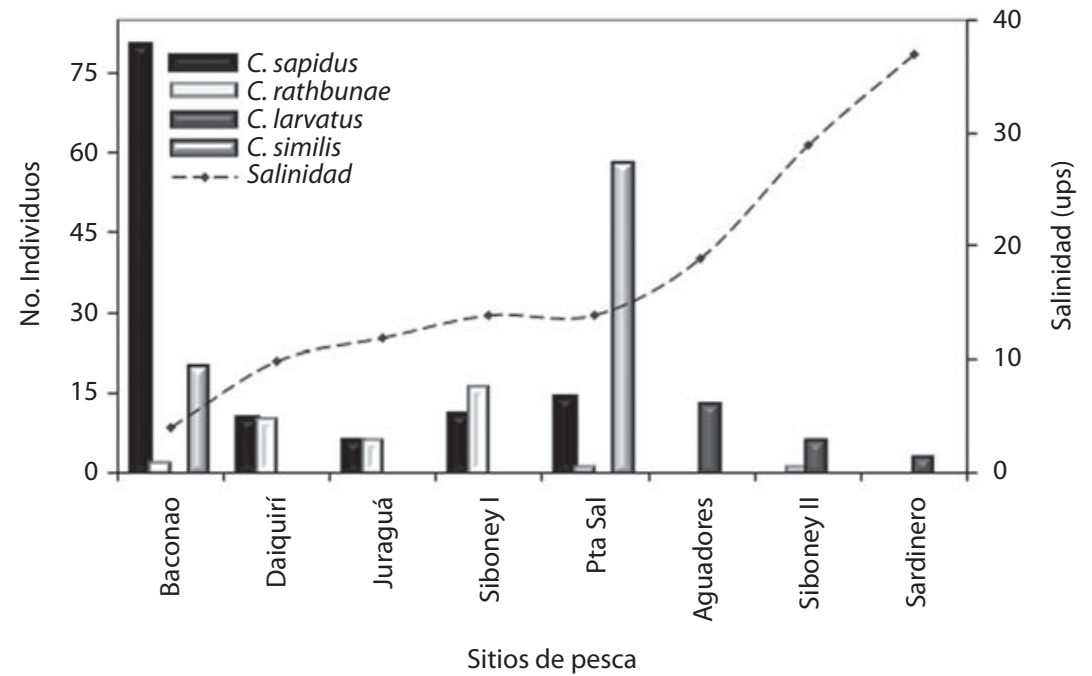

Fig. 1. Distribución de especies de Callinectes por sitios de captura y fluctuaciones de la salinidad.

Fig. 1. Distribution of Callinectes species by capture localities and salinity fluctuation.

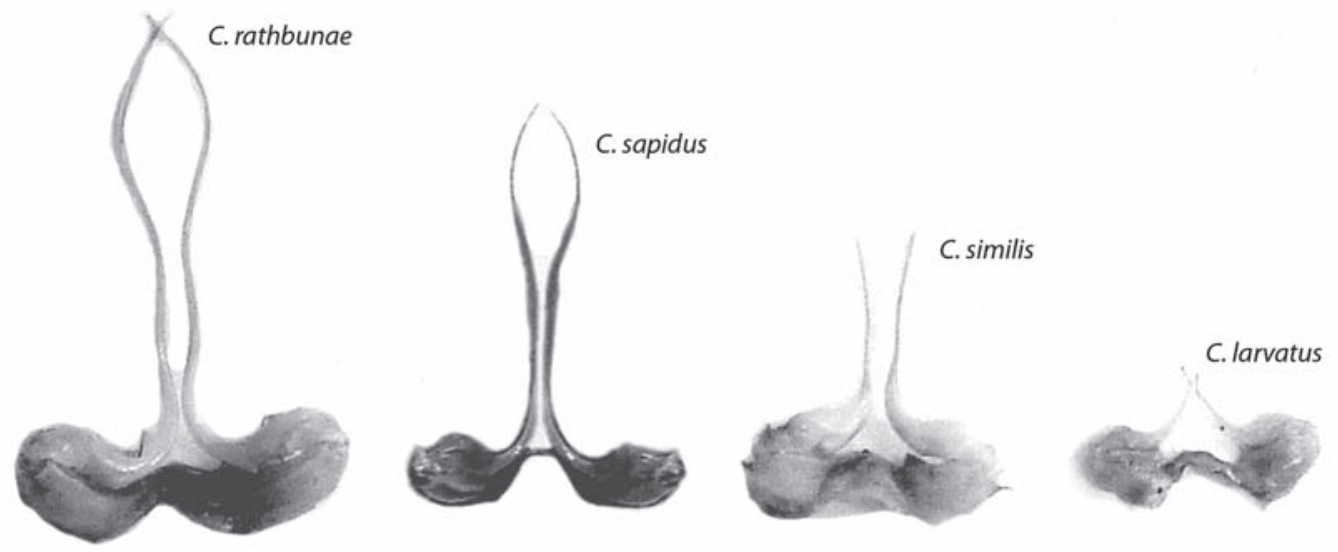

Fig. 2. Variación de la longitud y diseño de los gonopodios en las especies del género Callinectes identificadas en el litoral de Santiago de Cuba.

Fig. 2. Variation of length and design of gonopods in Callinectes species identified in Santiago de Cuba coastline.

recto y luego divergen para converger hacia el ápice, sin que exista cruzamiento. C. similis y C. larvatus tienen gonopodios más cortos, sin embargo, los de la primera tienen mayor longitud relativa y divergen hacia el ápice; en $C$. larvatus tienen un punto de cruce.

Esta característica, además del número de dientes frontales interorbitales, constituye un criterio relevante para la identificación de dichas especies.

Proporción entre sexos por especie y sitio de captura: En general se capturaron más machos que hembras. Los valores de $\mathrm{R}_{\mathrm{s}}$ por especie fueron significativamente diferentes $(\mathrm{p}<0.01): 6.20$ para C. rathbunae; 3.58 para 
C. similis; 1.40 para C. larvatus y 0.40 para

C. sapidus, destacando en todos los casos un sesgo en dichas proporciones a favor de los machos, excepto para C. sapidus. Las proporciones encontradas para $C$. rathbunae y $C$. similis se desvían significativamente de la del resto de las especies.

Es importante destacar que los menores valores de $\mathrm{R}_{\mathrm{s}}$ fueron encontrados en la especie más ampliamente distribuida.

El análisis por sitio de captura mostró valores de $R_{s}$ para Baconao de 0.44, único sitio donde la cantidad de hembras superó a los machos, con dominancia de C. sapidus; en Aguadores de 1.17, en Daiquirí de 1.22, en Sardinero y Juraguá de 2, en Punta Sal de 2.48, en Siboney II de 2.5 y en Siboney I fue de 8 , siendo esta última la estación que presentó menor proporción de hembras respecto a los machos $(\mathrm{p}<0.01)$.

En la figura 3 se presenta un gráfico donde se relacionan los valores de $\mathrm{R}_{\mathrm{s}}$ con la salinidad y la profundidad. En este sentido es importante señalar que los valores de $\mathrm{R}_{\mathrm{s}}$ fueron en la

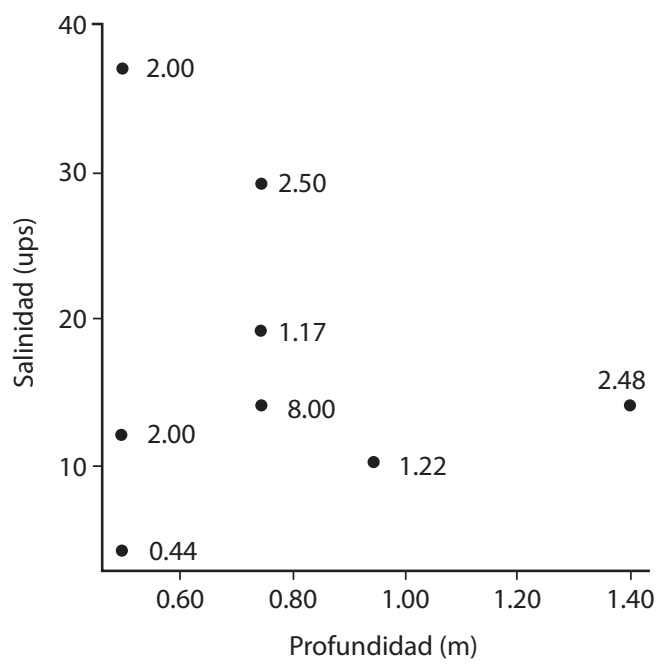

Fig. 3. Variación de los valores de $\mathrm{R}_{\mathrm{s}}$ con la profundidad $\mathrm{y}$ la salinidad en los sitios de captura.

Fig. 3. Variation of $\mathrm{R}_{\mathrm{s}}$ values in function of depth and salinity in capture localities. mayoría de los casos mayores que 1 , excepto en Baconao, donde la salinidad es menor de 10 ups y la profundidad de $0.50 \mathrm{~m}$.

Al parecer, en la zona litoral, la interacción entre la profundidad y la salinidad tiene una importante influencia sobre este parámetro.

Caracterización de ejemplares por especie: Los análisis morfométricos y pesajes permitieron determinar la longitud promedio (AC) y peso promedio (W) para cada especie: en $C$. sapidus el AC promedio fue de $110.57 \mathrm{~mm}$ (DS 21.55, $\mathrm{n}=121)$; y W $=84.46 \mathrm{~g}$ (SD 43.25, $\mathrm{n}=121)$; para $C$. rathbunae $\mathrm{AC}=115.50 \mathrm{~mm}$ (DS 14.94, $\mathrm{n}=36$ ) y $\mathrm{W}=140.44 \mathrm{~g}$ (DS 55.02, $\mathrm{n}=36)$. C. larvatus presentó menores valores de AC (76.04 mm, DS 10.88, $\mathrm{n}=22)$; y W (31.70 g, DS 14.67, $\mathrm{n}=22)$ y $C$. similis tuvo los valores mínimos $(\mathrm{AC}=59.77 \mathrm{~mm}$, DS 14.09, $\mathrm{n}=78)$ y $(\mathrm{W}=13.80 \mathrm{~g}$, DS 10.00, $\mathrm{n}=78)$; datos no evaluados hasta la fecha en las capturas del litoral. Los mayores ejemplares (AC>140 $\mathrm{mm}$ ) fueron capturados en Juraguá y Baconao, sitios que presentaron mejores condiciones de hábitat; estos corresponden a ejemplares de $C$. rathbunae y $C$. sapidus, especies que presentan las mayores tallas promedio, mejor distribución y mayor abundancia, respectivamente.

Contenido estomacal: Fueron analizados cualitativamente 257 estómagos y su contenido dividido en nueve categorías dietéticas, para las que se determinó la frecuencia de presencia (Cuadro 2). Los peces (0.75) y macroalgas (0.56) fueron las categorías más importantes en el litoral.

El MAD representa el (0.44), así como los granos de arena y sedimento, seguido por los moluscos bivalvos $(0.43)$, gasterópodos $(0.37)$ y anfípodos $(0.31)$ y restos de otros crustáceos (0.13) y plantas vasculares (0.12). En los contenidos estomacales de $C$. similis y $C$. larvatus no se encontraron restos de crustáceos. Asimismo los restos de plantas vasculares solo aparecen en C. sapidus.

Las categorías dietéticas no se mantienen por especie para los diferentes sitios de captura, variando notablemente de un sitio a otro. 


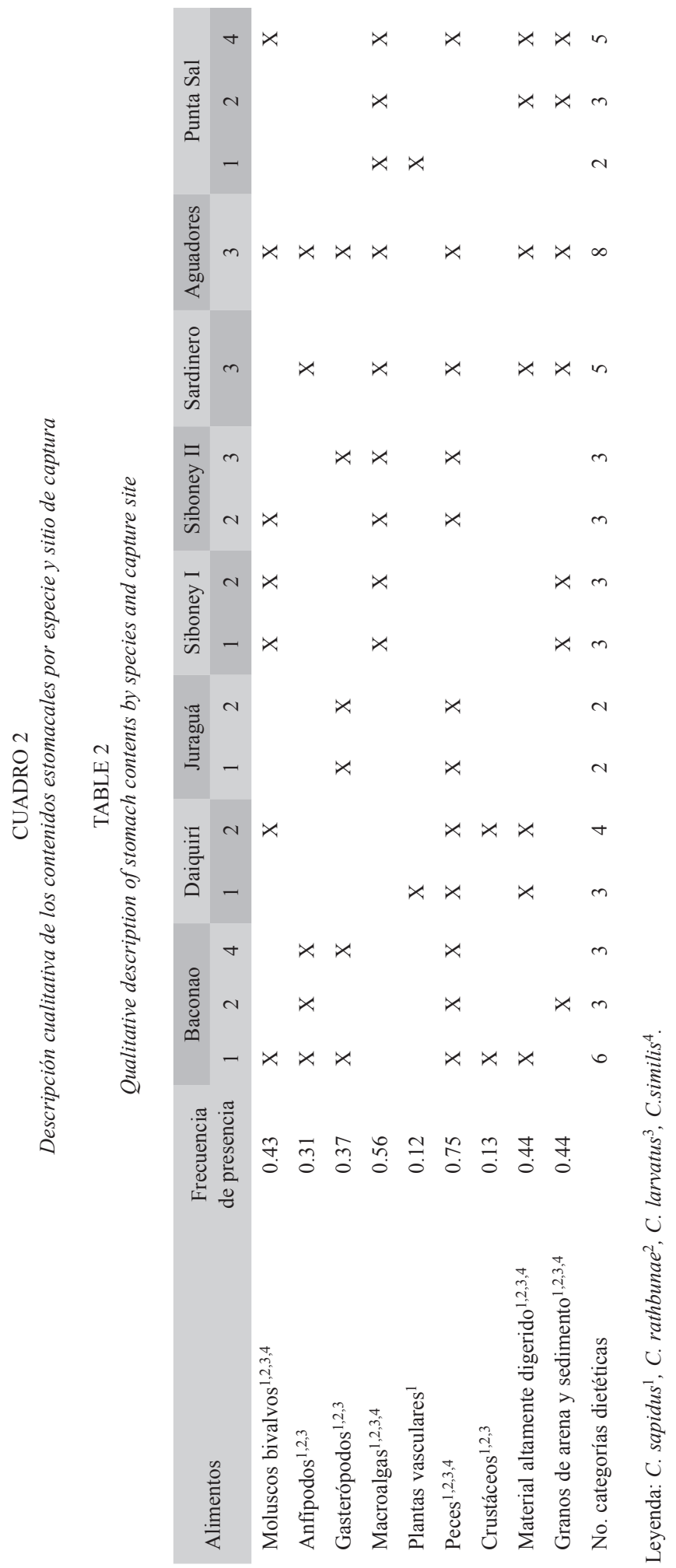




\section{DISCUSIÓN}

Los sitios de captura en el litoral resultaron ser muy heterogéneos, sin embargo, el género resultó estar ampliamente distribuido. Los sitios de captura con preferencia por los pescadores locales (Baconao y Aguadores) resultaron ser diferentes en cuanto a condiciones ambientales y especies presentes, siendo Aguadores no sólo un ecosistema de riesgo, atendiendo a la calidad del recurso dada por la calidad del hábitat, sino que constituye un sitio vulnerable en tanto parece existir una sola especie: C. larvatus. Baconao muestra excelentes condiciones ambientales, en este sitio se realizó la mayor captura y el número de hembras fue proporcionalmente mayor que el de machos, lo que apunta a una mayor disponibilidad del recurso.

De las cuatro especies identificadas en el litoral, $C$. rathbunae y $C$. similis no se incluyen en la lista actualizada de decápodos marinos cubanos; sin embargo Martínez- Iglesias y Gómez (1986) y Lalana y Ortiz (2000) citan la presencia de C. marginatus. Al respecto es importante considerar los criterios de Manning y Holthuis (1981) sobre la exclusión de C. marginatus del listado de especies del Altlántico Occidental; dichos autores refieren que existió sinonimia entre ambas especies y criterios taxonómicos sólidos permitieron separar a posteriori $C$. larvatus de C. marginatus, considerando esta última confinada a las costas africanas (Portunidae 2006).

Con este estudio se estarían identificando tres nuevas especies para Cuba (específicamente para la costa suroriental): $C$. rathbunae, $C$. similis y $C$. larvatus; si se considera la explicación precedente, serían dos.

En el caso de $C$. similis, si bien su distribución comprende la zona de estudio, hasta la fecha no había sido identificada en el litoral de Santiago de Cuba, situación similar a la de C. rathbunae, sólo incluida en los listados del Golfo de México, el Golfo de Venezuela y el Lago Maracaibo (Taissoun 1973). Estas omisiones pueden deberse fundamentalmente a la falta de estudios sistemáticos sobre Portúnidos en la costa suroriental; por otra parte, los listados realizados para Cuba refieren estudios entre los años 1903 a 1996 (Martínez-Iglesias y Gómez 1986, Lalana y Ortiz 2000).

El patrón de abundancia en la zona de estudio coincide con el encontrado por RochaRamírez et al. (1992) y Chazaro-Olvera et al. (2000) en el estado de Veracruz (Golfo de México); quienes explican que $C$. similis es la segunda especie más abundante, después de $C$. sapidus. En el caso de la distribución, un patrón similar ha sido referido por Van Engel (1958 a, b), Pounds (1964), Tagatz $(1965,1968)$ y Calderón (1996).

Las especies más ampliamente distribuidas son $C$. sapidus y C. rathbunae, sin embargo al analizar la dominancia, C. sapidus mostró un mayor índice en Baconao estación con los menores valores de salinidad y fondos arenofangosos, mientras que en tres de los sitios donde fue capturada, todos esteros con fondos fangosos y areno-fangosos, y salinidades entre 10 y 14 ups, convive sólo con C. rathbunae, con iguales índices de dominancia, excepto para Siboney I. Al parecer en estos biotopos ambas especies se han adaptado a convivir sin que se afecten sus poblaciones, manteniendo cierto equilibrio. En estos casos, al capturar ejemplares de gran talla, habría que considerar que estos pueden elegir áreas deseables en términos de parámetros abióticos y suministros alimenticios (Buchanan y Stoner 1988).

Respecto a la influencia de factores como la salinidad sobre los patrones de distribución, es bien conocida la alta tolerancia de C. sapidus, lo que le hace posible ocupar diversos hábitats. La salinidad es un factor importante en la distribución espacial de esta especie (Ortiz et al. 2007), abundante en bahías y desembocaduras de ríos, con una gran capacidad adaptativa; sin embargo, esta fue encontrada sólo en sitios con valores de salinidad entre 4 y 14 ups, lo que indica que su distribución espacial parece estar más relacionada con características particulares del hábitat.

C. sapidus puede encontrarse en salinidades que van desde 1.9 ups (Arreguín-Sánchez 1976) hasta 38 ups (Raz-Guzmán et al. 1986); 
Ortiz et al. (2007) explican su preferencia por salinidades de $12.73 \pm 1.6$ ups, concordando con Rocha-Ramírez et al. (1992). Otros factores como la textura de los fondos y la profundidad confirman que $C$. sapidus se encuentra en fondos de diferente textura y profundidades variables (Ortiz et al. 2007); sin embargo esta no fue capturada en playas con fondos arenosos y valores de salinidad por encima de 14 ups, lo que sin dudas evidencia una preferencia. $\mathrm{Su}$ dominancia fue constatada en capturas realizadas a menos de $1 \mathrm{~m}$ de profundidad. Coincidentemente, Rodríguez (1982) encontró en el Golfo de Guacanayabo, Cuba, una distribución fuertemente costera, específicamente para C. sapidus, con un gradiente de abundancia que disminuye a medida que aumenta la profundidad.

Calderón (1996), por su parte, explica que C. rathbunae muestra preferencia por salinidades bajas, lo que condiciona su distribución espacial. Ello justificaría que dicha especie no fue capturada en sitios con salinidades por encima de 30 ups, siendo mayoritaria en aquellos con valores entre 10 y 14 ups. Su dominancia en Siboney I sobre $C$. sapidus, llevó a la búsqueda de otros factores que pudieran condicionar su abundancia, encontrándose en este sitio, los más altos valores de Fe (Gómez et al. en prep). Se hace evidente además en esta especie, cierta preferencia por fondos fangosos.

C. larvatus fue capturada en sitios con salinidades superiores a los 19 ups y un sustrato preferiblemente arenoso, lo que indica una preferencia. Destaca además la baja diversidad en los sitios de captura de C. larvatus, donde habita prácticamente sola, excepto en Siboney II, donde fue capturado además, un ejemplar de C. rathbunae, hecho que pudo estar condicionado por la salinidad. En estaciones como esta podría existir una zonificación dada por los gradientes de salinidad, que minimicen la competencia por espacio y alimentos. Carmona-Suárez y Conde (2002) señalan que C. larvatus tiene una distribución muy restringida y vive sólo en sitios estuáricos. En el litoral de Santiago de Cuba, esta especie se asocia a sitios con valores de salinidad entre los 19 y
37 ups, muy antropizados (Aguadores, Siboney II y Sardinero), donde es dominante.

C. similis fue capturada en Baconao, pero resultó ser dominante en Punta Sal, a la mayor profundidad de captura $(1.40 \mathrm{~m})$, en un sitio muy resguardado, con fondos fangosos (cienofangosos), sedimentos de granulometría muy fina, asociado a manglares, con abundante alimento y cercanía a cursos de agua dulce. Sólo fue capturada en sitios con valores de salinidad de 4 y 14 ups. Considerando sus tallas, podrían ser las características del hábitat (refugio y alimento) las que determinan su distribución y dominancia.

Pinheiro et al. (1997) explican que los patrones de distribución de portúnidos en bahía Fortaleza (Brasil) están condicionados por la composición granulométrica de los sedimentos, lo que sin duda constituye un factor importante a considerar en la dominancia de $C$. similis.

Por otra parte, en Punta Sal destaca la presencia de jóvenes, los que se sabe, sobreviven donde hay pocos depredadores y mejor protección (Buchanan y Stoner 1988).

Existen criterios sobre una división de hábitat por sexo, determinada por la preferencia de determinados valores de salinidad (Hines et al. 1987, Ortiz et al. 2007), según el cual los machos se localizan en las zonas de menor salinidad y las hembras adultas migran hacia zonas de mayor salinidad, relacionado ello con los picos de apareamiento (William et al. 1990). Sin embargo, en este trabajo no se pudo establecer ni confirmar dicha correlación, debido fundamentalmente a la heterogeneidad de las estaciones y las diferencias entre las especies presentes. Quizás podría justificarse la prevalencia de machos por otro criterio, el de la profundidad, manejado por Chazaro-Olvera et al. (2000), en cuyas capturas se muestra la misma tendencia descrita en el presente trabajo; dichos autores explican la probable ubicación de hembras a mayor profundidad. Hay que tener en cuenta que las capturas en este estudio se realizaron hasta los $1.4 \mathrm{~m}$.

Si se analiza la diferencia del coeficiente $\mathrm{R}_{\mathrm{s}}$ en las estaciones de Siboney I y Punta Sal (8 y 2.48, respectivamente); para ambos sitios 
la salinidad fue la misma (14 ups); sin embargo, varió la profundidad a la que se capturaron los ejemplares ( 0.75 y 1.4 , respectivamente), existiendo un incremento de las hembras con el aumento de la profundidad, y por tanto, una disminución de $\mathrm{R}_{\mathrm{s}}$. Es importante entonces la interacción entre la salinidad y la profundidad para la definición de la proporción de machos y hembras, como regla general. Sin embargo, en Baconao, sitio que puede calificarse como un hábitat ideal, se capturaron más hembras en una zona donde coincidieron los menores valores de salinidad y menor profundidad, lo que demuestra que la preferencia por determinado hábitat está condicionada por las características particulares del sitio, y la interacción de diferentes parámetros; depende también de las especies presentes y el momento en que se realiza la captura.

$\mathrm{Al}$ analizar los valores de $\mathrm{R}_{\mathrm{s}}$ por especie, destaca la vulnerabilidad de $C$. rathbunae, con una proporción de machos muy alta respecto a las hembras. C. similis también mostró altos valores de $\mathrm{R}_{\mathrm{s}}$. Estos pudieron ser comparados con los citados por Carmona-Suárez y Conde (2002) para C. sapidus (0.70) y C. larvatus (2), en estaciones estuarinas de Ensena La Vela, Venezuela, superiores a los obtenidos en el presente estudio ( 0.40 y 1.40 , respectivamente).

En relación con la talla los mayores ejemplares corresponden a C. rathbunae $>C$. sapi$d u s>C$. larvatus $>C$. similis. Si bien el peso es un parámetro influido por factores biológicos y ecológicos, la altura del área metagástrica del carapacho y la longitud del carapacho, parecen tener valor para la identificación de las especies.

Es importante señalar que en todos los casos las tallas por especie son menores que las tallas promedio que refiere la literatura, lo que puede deberse a condiciones climáticas locales (elevadas temperaturas), la degradación de los hábitats y la presión post-captura. Es bien conocido que la calidad del hábitat es indudablemente muy importante cuando se considera la salud a largo plazo del recurso (Smithsonian Marine Station 2004).
En el caso de C. sapidus los valores citados son de 200 a $227 \mathrm{~mm}$ de AC (William 1974), mientras que en litoral de Santiago de Cuba, los promedios de AC son de $110.57 \mathrm{~mm}$ (DS 21.55, $\mathrm{n}=121$ ); para $C$. rathbunae los reportes son de 140 a $190 \mathrm{~mm}$ de AC (FAO 1996, Vrais Crabes 2005), mientras que el valor promedio obtenido fue $\mathrm{AC}=115.50 \mathrm{~mm}$ (DS 14.94, $\mathrm{n}=36)$. C. larvatus presentó valores de $\mathrm{AC}$ de $76.04 \mathrm{~mm}$ (DS 10.88, $\mathrm{n}=22$ ), mientras que se reportan valores de $\mathrm{AC}=95-142 \mathrm{~mm}$ (Vrais crabes 2005). C. similis presentó valores de AC $=59.77 \mathrm{~mm}(\mathrm{DS} 14.09, \mathrm{n}=78)$ los que difieren de los que reporta la literatura (95 a $122 \mathrm{~mm}$ ) (FAO 1996, Vrais crabes 2005).

Respecto a la dieta de estos cangrejos en el litoral, en los contenidos estomacales de todas las especies analizadas se identificaron restos de moluscos bivalvos, anfípodos, gasterópodos, macroalgas, peces, granos de arena, sedimento y MAD. Sin embargo, sólo en C. sapidus se encontraron restos de plantas vasculares y como hallazgo singular, no hay restos de crustáceos ni en C. similis, ni en C. larvatus, por lo que no hay evidencia de canibalismo en estas especies, lo que pudiera estar relacionado con la disponibilidad de alimentos y/o preferencias, que podrían tener cierta relevancia ecológica, considerando el papel de estos cangrejos en la cadena trófica.

En condiciones de suficiencia de alimentos las jaibas no son predadores directos de otros crustáceos; Paul (1981) refiere además que en estas circunstancias solo compiten por alimento y espacio, situación que puede darse en los sitios donde se capturó C. similis (Baconao y Punta Sal), debido a la abundancia y disponibilidad de alimento.

Perry y McIlwain (1986) explican que los cangrejos portúnidos son omnívoros bentónicos oportunistas. En efecto, las jaibas constituyen ejemplares activos y voraces, cuya dieta alimenticia puede estar basada en otros crustáceos, peces, gran variedad de moluscos, algas, anélidos y materiales en descomposición. La predación de estos regula las poblaciones de bivalvos marinos en aguas poco profundas, sobre los que muestran cierta preferencia, 
como fuentes de alimentación primaria, aunque los jóvenes más desarrollados y adultos incorporan a veces algún material vegetal como Ulva, Zostera y Spartina (Smithsonian Marine Station 2004); todo lo que depende de las condiciones del hábitat, donde cumplen una valiosa función desde el punto de vista ecológico (González y Aguilar 1984, Dittel et al. 2000).

De relevancia es el hecho de que los anfípodos son dominantes en el contenido estomacal de cangrejos colectados en sitios cercanos a lechos de macroalgas, donde son abundantes (Abele y King 1986); sin embargo, una observación importante es que no hay contenido de anfípodos en los jóvenes analizados.

Las categorías dietéticas no se mantienen por especie para los diferentes sitios de captura, variando notablemente de un sitio a otro, incluso las principales, que resultaron ser peces y macroalgas; por lo que se deduce que las jaibas comen no solo lo disponible, sino lo más abundante, con una conducta alimentaria más bien facilista, a pesar de estar catalogadas como depredadores activos.

En Baconao, por ejemplo, hay una rica ictiofauna y un pastizal cercano al sitio de captura, de aquí que las categorías más importantes fueran anfípodos y peces; en Daiquirí hay abundantes juveniles de peces y mucha materia vegetal en suspensión, debido a la abundante vegetación circundante, de aquí que los peces y el MAD fueran categorías dietéticas importantes. En Juraguá se confirmó la abundancia de peces juveniles y gasterópodos. En Siboney I hay parches de macroalgas y abundantes moluscos, identificados como categorías dietéticas relevantes; mientras que en Siboney II abundan peces provenientes de los arrecifes en parche cercanos a la playa y muy cerca del sitio de captura hay densos lechos de macroalgas. En el resto de los sitios se confirmó que los recursos más disponibles, aparecen en los contenidos estomacales, evidencia de que el hábitat define la conducta alimentaria.

El microhábitat es un factor determinante en la dieta de especies del género Callinectes (Laughlin 1982). Dittel (1993) afirma que existe una interacción entre la zona de muestreo y la estación o período del año, sugiriendo una variación temporal de la disponibilidad de alimentos. No obstante, es importante señalar que C. similis, independientemente del sitio donde se capturó, siempre incluyó peces en su dieta y C. larvatus, incorporó macroalgas y peces, por lo que pudiera haber ciertas preferencias relacionadas con la especie en cuestión.

\section{CONCLUSIONES}

El género Callinectes está representado por cuatro especies en el litoral de Santiago de Cuba: C. sapidus, C. rathbunae, C. similis y $C$. larvatus, constituyendo de manera general un recurso abundante de gran aceptación que sustenta la pesca local. Las especies más abundante y más ampliamente distribuida son C. sapidus y C. rathbunae, respectivamente. Existe de manera general una prevalencia de machos en todas las capturas realizadas, excepto para C. sapidus en Baconao, aspecto que debe ser considerado en los planes de manejo del recurso. Los sitios de captura incluidos en el estudio resultaron ser muy heterogéneos; la distribución y abundancia del género en el litoral está condicionada por la interacción de factores como la salinidad, profundidad, características de los fondos y otras propias del hábitat como su calidad de refugio y como proveedor de alimentos. Los ejemplares colectados fueron en todos los casos de menor talla que la citada en la literatura científica, lo que podría deberse a las características climáticas locales o a la contaminación existente en el litoral. De forma general, las especies del género Callinectes en el litoral de Santiago de Cuba presentan un espectro trófico diversificado con una estrategia alimentaria que implica consumir lo más abundante, lo que confirma la tesis de que la disponibilidad de alimentos en el microhábitat va a definir la conducta alimentaria.

\section{AGRADECIMIENTOS}

Este estudio fue desarrollado en el Laboratorio de Ecotoxicología del CNEA 
(Centro Nacional de Electromagnetismo Aplicado), en el marco de un proyecto universitario con el financiamiento del Programa de Cooperación al Desarrollo de la Universidad de Islas Baleares, a quien debemos el desarrollo exitoso de esta investigación. Agradecemos en especial a los pescadores de Baconao por su ayuda en el trabajo de campo.

\section{RESUMEN}

El objetivo del presente trabajo es el estudio del género Callinectes en la zona costera del municipio de Santiago de Cuba. Se realizó una recolecta de 257 ejemplares durante marzo de 2007 a abril de 2008, en ocho estaciones a lo largo de la costa. El género resultó estar ampliamente distribuido en el litoral y representado al menos por cuatro especies: C. sapidus, C. similis, C. rathbunae y C. larvatus, las dos últimas no incluidas en la lista publicada de crustáceos (Crustacea: Decapoda) cubanos. Las especies más abundantes resultaron $\operatorname{ser} C$. sapidus y $C$. similis. $C$. rathbunae fue la más ampliamente distribuida. El análisis del contenido estomacal permitió identificar nueve categorías alimentarias, siendo las más importantes los peces y las macroalgas. Las especies de Callinectes tienen un amplio espectro trófico; comen no sólo lo disponible, sino también lo más abundante.

Palabras clave: Callinectes, C. sapidus, C. similis, $C$. rathbunae y C. larvatus, cangrejo, Santiago de Cuba.

\section{REFERENCIAS}

Abele, L.G. \& W. Kim. 1986. An Illustrated Guide to the Marine Decapods Crustaceans of Florida. B. Mar. Sci. 32:807-822.

Adkins, G. 1972. A study of the blue crab fishery in Louisiana. La. Wildl. Fish. Comm. Oyster, Water Bottoms and Seafood Div. Tech. Bull. 3. Baton Rouge, La. 57 p.

Arreguín-Sánchez, S.F. 1976. Notas preliminares sobre las jaibas (Portunidae, Callinectes spp.) en las lagunas de Mandinga, pp. 159-171. In Mem. Rec. Pesq. Cost. Méx. 23-25 Nov. INP, Veracruz, México.

Baisre, J.A. 2004. La pesca marítima en Cuba. Científico Técnica, Ciudad de la Habana, Cuba. 372 p.

Branco, O.J., M.J. Lunardon-Branco, J.R. Verani, R. Schveitzerl, F.J. Souto \& W.G. Vale. 2002. Natural Diet of Callinectes ornatus Ordway, 1863 (Decapoda, Portunidae) in the Itapocoroy Inlet, Penha, SC, Brazil. Braz. Arch. Biol. Techn. 45: 35-40.
Buchanan, B.A. \& W.A. Stoner. 1988. Distributional patterns of blue crabs (Callinectes sp.) in a tropical estuarine lagoon. Estuaries 11: 231-239.

Calderón, J.N. 1996. Crecimiento de la jaiba azul Callinectes sapidus y de la jaiba prieta Callinectes rathbunae en la Laguna de Sontecomapan, Veracruz. Ph. D. Tesis. Facultad de Ciencias, Universidad Nacional Autónoma de México, México. 66 p.

Carmona-Suárez, C.A. \& J. Conde. 2002. Local distribution and abundance of swimming crabs (Callinectes spp. and Arenaeus cribrarius) on a tropical arid beach. Fish. B- NOOA. 100:11-25.

Chazaro-Olvera S., A. Rocha-Ramírez \& R. RománContreras. 2000. Observations on feeding, maturity, and fecundity of Callinectes similis Williams, 1966, on the central continental shelf off Veracruz, Gulf of Mexico. Crustaceana 73: 323-332.

Criales, M.M., C. Yeung, F. Amaya, A.C. López, D.L. Jones \& W.J. Richards. 2002. Larval supply of fishes, shrimps, and crabs into the nursery ground of the Ciénaga Grande de Santa Marta, Colombian Caribbean. Caribb. J. Sci. 38: 52-65.

Dittel, A.I. 1993. Cambios en hábitos de Callinectes arcuatus (Crustacea: Decapoda) en el Golfo de Nicoya, Costa Rica. Rev. Biol. Trop. 41:639-646.

Dittel, A.I., C.E. Epifanio, S.M. Schwalm, M.S. Fantle \& M.S. Fogel. 2000. Carbon and nitrogen sources for juvenile blue crabs Callinectes sapidus in coastal wetlands. Mar. Ecol. Prog. Ser. 194: 103-112.

García-Montes, J.F., L.A. Soto \& A. Gracia. 1988. Cangrejos Portúnidos del Suroeste del Golfo de México: aspectos pesqueros y ecológicos. An. Inst. Cienc. del Mar y Limnol. 15: 135-150.

Gómez, L.M, Y. Larduet \& N. Abrahantes. 2001. Contaminación y biodiversidad en ecosistemas acuáticos. El fitoplancton de la Bahía de Santiago de Cuba. Rev. Inv. Mar. 22: 191-197.

González, G. \& C. Aguilar. 1984. Ecología de las lagunas costeras de la región suroccidental de Cuba. Rev. Inv. Mar. 5: 127-171.

Gore, R.H. \& R.E. Grizzle. 1974. Studies on decapod Crustacea from the Indian River region of Florida. Chap. III. Callinectes bocourti A. Milne Edwards 1879 (Decapoda, Portunidae) from the Central East coast of Florida. Crustaceana 27: 306-308.

Hendricks, M.E. 1984. Estudio de la fauna marina y costera del Sur de Sinaloa, México III. Clave de Identificación de los cangrejos de la familia Portunidae (Crustacea: 
Decapoda). An. Inst. Cienc. del Mar y Limnol. 11: $1-246$.

Hendricks, M.E. 1995a. Cangrejos, 565-636 p. In W. Fischer, F. Krupp, W. Schneider, C. Sommer, K.E. Carpenter \& V.H. Niem (Eds.). Guía FAO para la identificación de especies para los fines de la pesca. Pacífico Centro-Oriental. Vol. 1. Plantas e Invertebrados. FAO. Roma, Italia.

Hendricks, M.E. 1995b. Checklist of brachyura crabs (Crustacea: Decapoda) from the eastern tropical Pacific. Bull. Inst. Roy. Sci. Nat. Belg. 65: 125-150.

Hines, A.H., R.N. Lipcius \& M. Haddon. 1987. Population dynamics and habitat partitioning by size, sex, and molt stage of blue crabs Callinectes sapidus in a subestuary of central Chesapeake Bay. Mar. Ecol. Prog. Ser. 36: 55-64.

Küçükgülmez, A. \& M. Çelik. 2008. Amino Acid Composition of Blue Crab (Callinectes sapidus) from the North Eastern Mediterranean Sea. J. Appl. Biol. Sci. 2: 39-42.

Lalana, R. \& M. Ortiz. 2000. Lista actualizada de los crustáceos decápodos de Cuba. Rev. Inv. Mar. 21: 33-44.

Laughlin, R.A. 1982. Feeding habits of the blue crabs, Callinectes sapidus Rathbun, in the Apalachicola estuary (North Florida USA). Ph.D. Dissertation. Flo. State Univ. Tallahassee, Florida, EEUU.

Manning, R.B. \& L.B. Holthuis. 1981. West African Brachyuran Crabs (Crustacea: Decapoda). Smithsonian Contributions to Zoology 306.

Markham, J.C., F.E. Donath-Hernández, J.L. VillalobosHiart \& A.C. Díaz-Barriga. 1987. Notes of the shallow-water marine crustacean of the Caribbean coast of Quintana Roo, México. Anal. Inst. Biol. 61: 405-446.

Martínez-Iglesias, J.C. \& O. Gómez. 1986. Los crustáceos decápodos del Golfo de Batabanó. Brachiura. Poeyana 332: 1-91.

Moncada, F.G. \& O. Gómez. 1980. Algunos aspectos biológicos de tres especies del género Callinectes (Crustacea, Decapoda). Rev. Cub. Inv. Pesq. 5:1-35.

Norse, E.A. 1972. Preliminary study of six Jamaican blue crabs, genus Callinectes (Decapoda: Portunidae). Atoll. Res. Bull. 152: 5.

Norse, E.A. 1977. Aspects of the zoogeographic distribution of Callinectes (Decapoda: Portunidae). B. Mar. Sci. 27: 440-447.
Norse, E.A. 1978. An experimental gradient analysis: Hyposalinity as an 'upstress' distributional determinant for Caribbean portunid crabs. Biol. Bull. 155: 586-598.

Ortiz-León H.J., A. Navarrete \& E. Sosa. 2007. Distribución espacial y temporal del cangrejo Callinectes sapidus (Decapoda: Portunidae) en la Bahía de Chetumal, Quintana Roo, México. Rev. Biol. Trop. 55: 235245.

Paul, R.K.G. 1981. The Development of a Fishery for Portunid Crabs of the Genus Callinectes (Decapoda, Brachyura) in Sinaloa, México. Final Report. Overseas Development Administration, Londres, Inglaterra. $78 \mathrm{p}$.

Perry, H.M. \& T.D. McIlwain. 1986. Blue crab-species profiles: Life histories and environmental requirements: U.S. Fish and Wildlife Service, Slidell, La, FWS/ OBS-82/11.55, $21 \mathrm{p}$.

Pinheiro, M.A.A., A. Fransozo \& M.L. Negreiros-Fransozo. 1997. Dimensionamento e sobreposição de nichos dos portunídeos (Decapoda, Brachyura), na Enseada da Fortaleza, Ubatuba, São Paulo, Brasil. Rev. Bras. Zool. 14: 371-378.

Pounds, S. 1964. The crabs of Texas. Coastal Fisheries. Texas Parks and Wildlife Department. Austin, Texas. Bull. 43: 57 p.

Ramírez, M.S. \& I. Hernández. 1988. Investigación biológico-pesquera para la obtención de jaiba suave Callinectes spp. en la Laguna de Alvarado, Veracruz. ENEP Iztacala, Ph.D. Tesis. Universidad Nacional Autónoma de México, México. 97 p.

Ramos, P.J.L., A.A. Granados \& O. Lárraga. 1998. La pesquería de las jaibas del género Callinectes (Decapoda: Portunidae) en el suroeste del Golfo de México. Univ. Cienc. 14: 65-78.

Raz-Guzmán, A., A. Sánchez, L. Soto \& F. Álvarez. 1986. Catálogo ilustrado de cangrejos braquiuros y anomuros de la laguna de Términos, Campeche (Crustacea: Brachyura: Anomura). Inst. Biol. UNAM 57: 383.

Robles, R., C. Schubart, J. Conde, C. Carmona-Suárez, F. Álvarez, J. Villalobos \& D. Felder. 2007. Molecular phylogeny of the American Callinectes Stimpson, 1860 (Brachyura: Portunidae), based on two partial mitochondrial genes. Mar. Biol. 150: 1265-1274.

Rocha-Ramírez, A., S. Chazaro \& P.M. Muller. 1992. Ecología del género Callinectes (Brachyura: Portunidae) en seis cuerpos de agua costero del Estado de Veracruz, México. An. Inst. Cienc. del Mar y Limnol. 19: 33-41. 
Rodríguez, M.J. 1982. Posibilidades de aprovechamiento de la jaiba azul (Callinectes sapidus, Rathbun) de la fauna de acompañamiento de la pesquería de camarón de Manzanillo. Rev. Cub. Inv. Pesq. 7:46-52.

Román-Contreras, R. 1986a. Comportamiento nictemeral de crustáceos decápodos en la boca de Estero Pargo, Laguna de Términos, Campeche, México. Anal. Inst. Cienc. del Mar y Limnol. 13: 149-158.

Román-Contreras, R. 1986b. Análisis de la población de Callinectes spp. (Decapoda: Portunidae) en el sector occidental de la Laguna de Términos, Campeche, México. Anal. Inst. Cienc. del Mar y Limnol. 13: 315-322.

Rosas, V.C. \& A. Sánchez. 1994. Fisiología de la adaptación de los crustáceos decápodos al ambiente lagunar estuarino, p. 305-332. In G. de la Lanza \& C. Cáceres. (Eds.) Lagunas costeras y el litoral mexicano. Universidad Autónoma de Baja California Sur, Baja California, México.

Rosas, V.C. \& E. Lázaro-Chávez. 1986. Efecto de las variaciones de salinidad sobre la tasa respiratoria de dos especies de jaibas: Callinectes sapidus y Callinectes rathbunae, en la laguna de Tamiahua, Veracruz, México. Rev. Invest. Mar. 7: 71-79.

Tagatz, M.E. 1965. Growth of juveniles' blue crabs, Callinectes sapidus Rathbun, in St. John's River, Florida. U. S. Fish. Fish. B- NOOA. 67: 281-288.

Tagatz, M.E. 1968. Biology of the blue crab, Callinectes sapidus Rathbun, in St. Johns River, Florida. U.S. Fish. B- NOOA. 67:17-33.

Taissoun E. 1973. Estudio comparativo, taxonómico y ecológico entre los cangrejos (Decapoda Brachyura Portunidae), Callinectes maracaiboensis (nueva especie), C. bocourti (Milne-Edwards) y C. rathbunae (Contreras) en el Golfo de Venezuela, Lago de Maracaibo y Golfo de México. Bol. Cent. Invest. Biol. 6: 77 p.
Tsai D.E., H. Chen H \& C. Tsai. 1984. Total lipid and cholesterol content in the blue crab, Callinectes sapidus Rathbun. Comp. Biochem. Phys. 78: 27-31.

Valdés, E., J.I. Noyola \& P. Machado. 1994. Resultado de la pesca exploratoria de jaiba en la plataforma cubana durante 1990. Rev. Cub. Inv. Pesq. 18: 16-20.

Van Engel, W.A. 1958a. The blue crab and its fishery in Chesapeake Bay. Part 1. Reproduction, early development, growth and migration. Commer. Fish. Rev. 20: 20.

Van Engel, W.A. 1958b. The blue crab and its fishery in Chesapeake Bay. Part 2. Types of Gear for Llard Crab Fishing. Commer. Fish. Rev. 24: 1-10

Williams, A.B. 1974. The swimming crabs of the genus Callinectes (Decapoda: Portunidae) Texas USA. Fish. B- NOOA. 72: 685-798.

Williams, A.B. 1984. Shrimps, lobsters and crabs of the Atlantic coast of the Eastern United States, Maine to Florida. Smithsonian Inst. Washington, D.C., EEUU. 550 p.

Williams, H.A., L.D. Coen \& M.S. Stoelting. 1990. Seasonal abundance, distribution and habitat selection of Callinectes sapidus (Rathbun) in the Northern Gulf of Mexico. J. Exp. Mar. Biol. Ecol. 140: 107-119.

\section{REFERENCIAS DE INTERNET}

FAO. 1996. Breviario de la pesquería de jaiba del Golfo de México y Mar Caribe. (Consultado: 3 marzo 2008, www.inp.sagarpa.gob.mx/Publicaciones/sustentabilidad/Golfo/JAIBAGOLFO.pdf).

Portunidae. 2006. (Consultado: enero 10, 2006, www.fao. org/docrep/fao/009/y4160e/y4160e23.pdf).

Smithsonian Marine Station. 2004. (Consultado: enero 23, 2004, http://www.sms.si.edu/irlspec.htm).

Vrais Crabes. 2005. (Consultado: febrero 3, 2005, www. fao.org/docrep/fao/008/t0438f/T0438F40.pdf). 